\title{
Isolation and Molecular Characterization of Feline Herpesvirus 1 from Naturally Infected Korean Cats
}

\section{Dong-Kun Yang*, Ha-Hyun Kim, Yu-Ri Park, Jae Young Yoo, Sung-Suk Choi, Yeseul Park, Sungjun An, Jungwon Park, Jongho Kim, Heui-Jin Kim, Jienny Lee, Bang-Hun Hyun}

Viral Disease Research Division, Animal and Plant Quarantine Agency, MAFRA, Gimcheon 39660, Republic of Korea

\author{
Corresponding \\ Dong-Kun Yang, Ph D, DVM \\ Animal and Plant Quarantine Agency, \\ 177 Hyeoksin 8-ro, Gimcheon-si, \\ Gyeongsangbuk-do 39660, Republic of \\ Korea \\ Phone : +82-54-912-0785 \\ Fax : +82-54-912-0812 \\ E-mail : yangdk@korea.kr
}

Received : July 22, 2020

Revised : December 6, 2020

Accepted : December 7, 2020

No potential conflict of interest relevant to this article was reported.

Copyright (C) 2020 Journal of Bacteriology and Virology

(C) This is an Open Access article distributed under the terms of the Creative Commons Attribution Non-Commercial License

(http://creativecommons.org/ license/by-nc/3.0/).
Feline herpesvirus type 1 (FHV-1) causes respiratory and ocular disease in cats. Although isolates of FHV-1 circulating in cats have been reported worldwide, Korean FHV-1 isolates and their features have not been reported thus far. We aimed to investigate the biological and molecular characterization of two FHV-1 isolates based on the nucleotide sequence of thymidine kinase $(T K)$ and glycoprotein $B(g B)$ gene. In total, 48 samples from 12 cats were prepared for virus isolation. For the diagnosis, virus isolation, indirect fluorescence assay (IFA), electron microscopy (EM), and polymerase chain reaction (PCR) and for the molecular characterization, cloning and sequencing were used. Based on many methods such as virus isolation with specific cytopathic effects, IFA, EM, and PCR, two isolates were confirmed as FHV-1 and they showed the highest viral titer $\left(10^{8.3}\right.$ to $10^{8.5}$ $\mathrm{TCID} 50 / \mathrm{mL}$ ) in the Crandell-Rees Feline Kidney cells at $48 \mathrm{~h}$ after inoculation, but did not grow in MDCK and Vero cells. The nucleotide and amino acid sequences of the full $T K$ and $g B$ gene of FHV191071 and FHV191072 isolates were determined and compared with those of other herpesvirus strains. Two isolates possessed the same nucleotide sequences belonging to $\mathrm{FHV}-1$ group and had the highest similarity (99.9\%) with the KANS-02 strain, which was isolated from shelter in USA in 2016. Two isolates were confirmed as FHV-1 and they will be a useful basic resource for evaluating current FHV-1 vaccine and developing diagnostic tools.

Key Words: Feline herpes virus 1, Virus isolation, Genetic characterization

\section{INTRODUCTION}

Feline herpesvirus 1 (FHV-1) belongs to the genus Varicellovirus, subfamily Alphaherpesvirinae in the family Herpesviridae. The FHV-1 genome has approximately 135,800 nucleotides including unique long (UL) and unique short (US), and terminal and inverted repeat long segments, which contain 78 open reading frames and have a $\mathrm{G}+\mathrm{C}$ content of approximately $45 \%(1,2)$. FHV-1 has 23 virion-associated proteins and its envelope consists of 13 glycoproteins: UL53 (gK), UL49.5 (gN), UL44 (gC), UL43, UL27 (gB), UL22 (gH), UL20, UL10 (gM), UL1 (gL), US4 (gG), US6 (gD), US7 ( $\mathrm{gl}$ ), and US8 (gE) (2). Four viral proteins have been identified as potential virulence factors: $g E, g C$, thymidine kinase (TK, UL23), and serine/threonine protein kinase (US3) (3). Glycoprotein B (gB, UL 27), a vital component of the surface of FHV-1 that is known to induce a protective immune response, is used as an enzymelinked immunosorbent assay antigen for detection of FHV-1 antibodies (4). 
Clinical signs of FHV-1 infection in cats are fever, nasal and ocular discharge, conjunctivitis, keratitis, and pneumonia; sometimes, abortion and fetal death can occur (5). Following the reduction in maternal antibodies, 6-9-week-old cats are more likely to be infected with FHV-1. Infections in cats have been reported in many countries (6-9). Transmission typically occurs via the oronasal route, following contact with ocular discharge from acutely infected cats (10). Similar to latent infection reported in other herpesviruses, trigeminal ganglion FHV-1 infection can be reactivated by strong stress and immunosuppression, resulting in infectious virus shedding (11).

The diagnosis of FHV-1 infection is based on virus isolation with cell cultures, pathological findings, the direct fluorescent antibody test, polymerase chain reaction $(P C R)$, real-time $P C R$, and serological assays $(4,12,13)$. Virus neutralizing antibody titers measured with the virus neutralization assay are used to monitor sero-epidemiological status or to evaluate the immunogenicity of vaccination (14). Among the various diagnostic methods, virus isolation assesses the efficacy of trial feline vaccines and is used to establish serological diagnostic assays. It is also useful when developing new vaccines for FHV-1. Because FHV-1 has a global distribution, many FHV-1 strains have been isolated from cats and tigers $(6,7,15)$. Although FHV-1 infection was examined in cats at a Korean animal shelter in 2008 (9), there has been no report of the successful isolation of Korean FHV-1 or its genetic features.

In this study, we isolated two FHV-1 isolates from naturally infected cats by Crandell-Rees feline kidney (CRFK) cell culture. They were designated FHV191071 and FHV191072; notably, they were propagated and passaged five times in the cells. In addition, we examined the properties of both isolates and determined the nucleotide sequences of the $T K$ and $g B$ genes of both isolates to evaluate their genetic relationships. The results of this study increase our knowledge of the biological and molecular features of Korean FHV-1 isolates.

\section{MATERIALS AND METHODS}

\section{Samples and preparation}

In this study, 48 samples of lung, spleen, brain, kidney, liver, and feces were prepared from 12 cats that had been sent to the Animal and Plant Quarantine Agency (APQA) for disease diagnosis in 2019. One gram of each sample was placed in a mortar and ground with a small amount of sea sand; $9 \mathrm{~mL}$ of Dulbecco's modified Eagle's medium (DMEM) was added to make a $10 \%$ homogenate. The homogenates were centrifuged at 2,500 $\times g$ for $15 \mathrm{~min}$ to remove tissue debris; the supernatants were filtered through a $0.45-\mu \mathrm{m}$ syringe filter to avoid bacterial infection.

\section{Virus isolation, growth kinetics, and titration}

The CRFK (CRFK, ATCC, CCL-94) cells used for virus isolation were cultured in DMEM supplemented with two antibiotics (penicillin and streptomycin) and one antimycotic (amphotericin B), as well as 10\% fetal bovine serum (FBS). Confluent CRFK cells in 24-well plates were washed twice with phosphate-buffered saline (PBS, pH 7.2), then incubated with $200 \mu \mathrm{L}$ of each filtered sample at $37^{\circ} \mathrm{C}$ for $1 \mathrm{~h}$. Subsequently, the liquid in the 24 -well plates was removed and $1 \mathrm{~mL}$ of fresh DMEM containing 3\% FBS was added to each well. Cells were incubated in a $5 \% \mathrm{CO}_{2}$ incubator at $37^{\circ} \mathrm{C}$ for 7 days. If the CRFK cells in the second passage did not show any cytopathic effects (CPE) after 7 days, the samples were considered negative for virus isolation. The supernatant in wells of cells with CPE was harvested and inoculated into newly prepared CRFK cells in a 6-well plate for the second passage. The growth kinetics of the FHV191071 and FHV191072 isolates were examined twice to determine the most rapid growth time. Briefly, CRFK cells grown in $25-\mathrm{cm}^{2}$ flasks were inoculated with isolates containing $10050 \%$ tissue culture infectious dose $\left(\mathrm{TCID}_{50} / \mathrm{mL}\right)$ and harvested at 12 -h intervals for $84 \mathrm{~h}$. After three continuous freeze-thaw processes, the viral titers of both isolates were determined based on the presence of typical CPE as follows: $100 \mu \mathrm{L}$ of each virus diluted 10-fold was distributed in 96-well microplates; $100 \mu \mathrm{L}$ of fresh medium 
containing $2 \times 10^{5}$ CRFK cells and 10\% FBS was added to the wells of the microplate. CRFK cells were observed for 5 days post-inoculation for typical CPE. Viral titers calculated by the Spearman-Karber method were expressed as the TCID ${ }_{50} / \mathrm{mL}$. To identify the optimal cells for viral propagation, both isolates were inoculated into six cell types in $25-\mathrm{cm}^{2}$ flasks: CRFK, Fcwf-4 (ATCC, CRL2728), BHK-21 (ATCC, CCL-10), A72 (ATCC, CRL-1542), Madin-Darby canine kidney (MDCK; ATCC, CCL-34), and Vero (ATCC, CCL81). After incubation for 5 days, each flask was frozen and thawed three times. Viral titers of the centrifuged solutions were measured using the method described above.

\section{Immunofluorescent assay}

CRFK cells infected with isolates in 96-well microplates were fixed with cold $80 \%$ acetone at $-20^{\circ} \mathrm{C}$ for $15 \mathrm{~min}$. The cold acetone was then discarded and the cells were reacted with a mouse anti-feline herpesvirus type 1 antibody (Bio-Rad, Hercules, CA, USA) at $37^{\circ} \mathrm{C}$ for $1 \mathrm{~h}$; they were then stained with fluorescent isothiocyanate-conjugated goat-anti-mouse IgG + IgM antibodies (KPL Laboratories, Gettysburg, PA, USA) diluted 200-fold in PBS under the conditions described above. Subsequently, the cells were washed with PBS twice, then air-dried and examined under a 200x fluorescence microscope (TE2000-U, Nikon Instruments, Tokyo, Japan). Specific fluorescence of CRFK cells with CPE was considered to indicate FHV-1 infection.

\section{Electron microscopy (EM)}

Isolates were propagated in CRFK cells, then concentrated and purified for photography of intact viral particles as follows: CRFK cells infected with the FHV191071 and FHV191072 isolates were harvested at $48 \mathrm{~h}$ after inoculation, in accordance with the growth kinetics, then frozen and thawed three times. Polyethylene glycol-precipitated pellets were dissolved in TNE buffer ( $100 \mathrm{mM}$ Tris-Cl, $100 \mathrm{mM} \mathrm{NaCl}$, and $1 \mathrm{mM}$ EDTA, pH 7.6) at $5 \%$ of the original volume. The concentrated samples were then subjected to sucrose purification, placed on Formvar-coated grids, and stained negatively with $1 \%$ uranyl acetate. To identify viral particles in infected cells, CRFK cells were prepared and inoculated with each isolate. At 30 h post-inoculation, cells were harvested and fixed with $2.5 \%$ glutaraldehyde and $1 \%$ osmium tetroxide solution in PBS. The fixed cells were embedded in resin and cut thinly with a microtome for staining; sliced cells were stained with uranyl acetate and lead citrate. Virus particles in the two isolates on the grid and sliced sections were examined under a Hitachi 7100 electron microscope (Tokyo, Japan).

\section{Hemagglutination (HA) assay}

For the HA assay, CRFK cells infected with the isolates were serially diluted two-fold with $50 \mu \mathrm{L} \mathrm{PBS} \mathrm{(pH} \mathrm{7.2);50} \mathrm{\mu L} \mathrm{of} 0.5 \%$ erythrocytes obtained from cats, guinea pigs, and mice were then added and incubated at 4,22 , and $37^{\circ} \mathrm{C}$ for $1 \mathrm{~h}$. The $\mathrm{HA}$ titers were expressed as the reciprocal of the highest dilution of $\mathrm{FHV}-1$ that demonstrated an $\mathrm{HA}$ reaction.

\section{$P C R$, sequencing, and phylogenetic analysis}

DNA was extracted from both isolates using a DNA extraction kit (Bioneer, Daejeon, Korea), in accordance with the manufacturer's instructions. DNA attached to the column was eluted with $50 \mu \mathrm{L}$ of elution buffer provided in the kit. PCR was performed for diagnosis and cloning. Table 1 lists the primers for detection of FHV-1 and amplification of the full TK and $g B$ genes. The reaction mixture for One-Step PCR kits (Bioneer) consisted of $5 \mu \mathrm{L}$ denatured DNA, $1 \mu \mathrm{L}$ each primer (50 $\mathrm{pmol} / \mathrm{LL}$ ), and $43 \mu \mathrm{L}$ distilled water. A thermal cycler (Analytik Jena, Jena, Germany) was used to perform 40 cycles of $95^{\circ} \mathrm{C}$ for $45 \mathrm{~s}, 50^{\circ} \mathrm{C}$ for $45 \mathrm{~s}$, and $72^{\circ} \mathrm{C}$ for $45 \mathrm{~s}$, with a final 10 -min extension at $72^{\circ} \mathrm{C}$. Approximately $5 \mu \mathrm{L}$ of each PCR product was loaded on a $2.0 \%$ agarose gel and subjected to electrophoresis for $30 \mathrm{~min}$. 
Table 1. Primers used for polymerase chain reaction analysis to amplify the thymidine kinase and glycoprotein B genes of the two isolates

\begin{tabular}{|c|c|c|c|c|}
\hline Designation & Oligonucleotide $\left(5^{\prime} \rightarrow 3^{\prime}\right)$ & Target gene & Size $(b p)$ & Remarks \\
\hline FHVDF1 & ATCTCGAGATCTACTTGACATGCATG & \multirow{2}{*}{ TK } & \multirow{2}{*}{519} & \multirow{2}{*}{ Diagnosis } \\
\hline FHVDR1 & GATGCATCATCGGTAAACCAGTCAAC & & & \\
\hline FHVTKF1 & ACTGCGTCGTTGTTCACGTTTC & \multirow{4}{*}{ TK } & 620 & \multirow{4}{*}{ Sequencing } \\
\hline FHVTKR1 & GGTTCTCGTGGAAGTGTTGC & & 20 & \\
\hline FHVTKF2 & GCAACACTTCCACGAGAACC & & \multirow{2}{*}{670} & \\
\hline FHVTKR2 & GGGGGGTAATAGGGAACACCA & & & \\
\hline FHVgBF1 & CAGGTACCCAGATCTGCCCA & \multirow{4}{*}{$g B$} & \multirow{2}{*}{889} & \multirow{6}{*}{ Sequencing } \\
\hline FHVgBR1 & CAGAGGTCCCAGAGTGGTGA & & & \\
\hline FHVgBF2 & GACGAGGATCCCAGAGAACT & & 1100 & \\
\hline FHVgBR2 & ATTGTGTTACGGCGACGGCA & & & \\
\hline FHVgBF3 & GAAATGTTGAGTCGGATAGCCAC & \multirow{2}{*}{\multicolumn{2}{|c|}{1194}} & \\
\hline FHVgBR3 & GAGATGGTCTGGTTGGTTAGAC & & & \\
\hline
\end{tabular}

* $T K$, thymidine kinase; $g B$, glycoprotein $B$

Five PCR products of partial $T K$ and $g B$ genes for cloning were purified by gel extraction; each product was ligated into the $\mathrm{pGEM}^{\circledR}$-T Easy vector system (Promega, Madison, WI, USA), in accordance with the manufacturer's instructions. Selected white colonies grew in Luria-Bertani broth containing $50 \mu \mathrm{g} / \mu \mathrm{L}$ ampicillin. Plasmid DNA was extracted from several white Escherichia coli (DH5 $\alpha$ ) colonies; each DNA insert in the plasmid was identified by ECoR1 digestion (Bioneer). Sequencing was performed by Macrogen (Daejeon, Korea). Each DNA sequence of both strands was cross-checked using the universal primer sets SP6 and T7 to verify the sequences. Two or three partial nucleotide sequences were linked to confirm the full $T K$ and $g B$ gene sequences. Nucleotide sequence analysis was performed based on the full $T K$ and $g B$ genes of the FHV191071 and FHV191072 isolates, as well as other herpesviruses obtained from GenBank. The presumptive amino acid sequences of the TK proteins of the two isolates and the KANS-02 strain were compared with Clone Manager Basic ver. 9 (Sci-Ed Software, Denver, CO, USA). Two phylogenetic trees were constructed using MEGA ver. 7.0.20 (http://megasoftware.net). The bootstrap method with 1,000 replicates was used to assess the reliability of the phylogenetic trees.

\section{Mouse inoculation test}

A mouse experiment was performed at APQA, Korea using protocols approved by the experimental animal ethics committee (approval number: 2020-431). To evaluate the virulence of the FHV191071 and FHV191072 isolates, 18 four-week-old Balb/c mice (OrientBio, Seongnam, Korea) were divided into three groups. Two groups of six mice each were peritoneally inoculated with $10^{8.0} \mathrm{TCID}_{50} / \mathrm{mL}$ of the FHV191071 and FHV191072 isolates. The third group served as a control; mice in that group received no treatment. Mice were observed for clinical signs (e.g., itching, depression, and loss of appetite and body weight) for 10 days.

\section{RESULTS}

\section{Isolation and biological characterization of the isolates}

Of the CRFK cells inoculated with the 48 samples, only two samples from two cats exhibited specific CPE, including cell 
aggregation, detachment, and cell rounding (Fig. 1A, B). The samples with distinct CPE were named FHV191071 and FHV191072, respectively; CRFK cells infected with each isolate were fixed in cold acetone and reacted with mouse monoclonal antibodies against FHV-1. As shown in Fig. 1D and E, specific fluorescence was observed in CRFK cells infected with FHV191071 and FHV191072. Growth kinetics were examined to determine when the isolates exhibited the highest titer. As shown in Fig. 2A, the highest viral titers $\left(10^{8.3}\right.$ and $\left.10^{8.5} \mathrm{TCID}_{50} / \mathrm{mL}\right)$ were found when viruses were harvested $48 \mathrm{~h}$ after inoculation. Both isolates were inoculated into six cell types to assess cell tropism. Of the six cell types, both isolates exhibited the highest viral titers (range, $10^{8.4}$ to $0 \mathrm{TCID}{ }_{50} / \mathrm{mL}$ ) in CRFK cells and did not show any CPE in MDCK or Vero cells (Fig. 2B). HA assays were performed with three types of erythrocytes; neither isolate was able to agglutinate erythrocytes from cats, guinea pigs, or mice (data not shown). Based on the FHV191071 and FHV191072 growth kinetics, virus

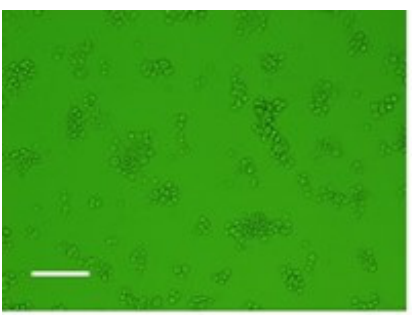

A

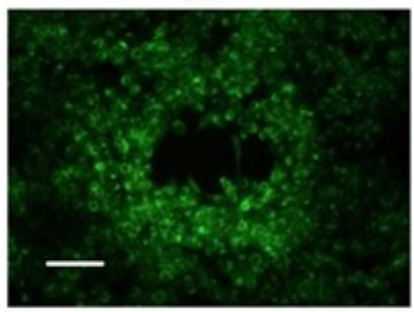

$\mathrm{D}$

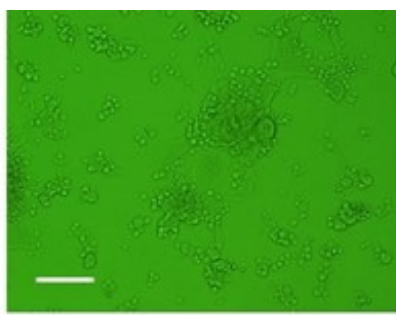

B

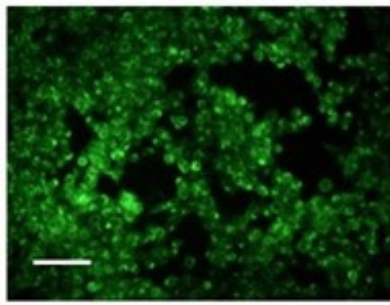

$\mathrm{E}$

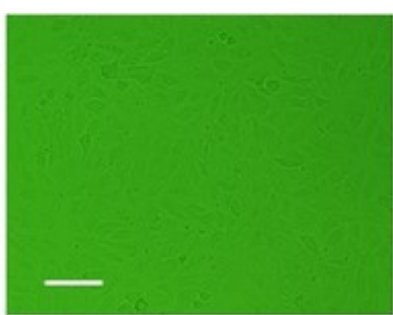

$\mathrm{C}$

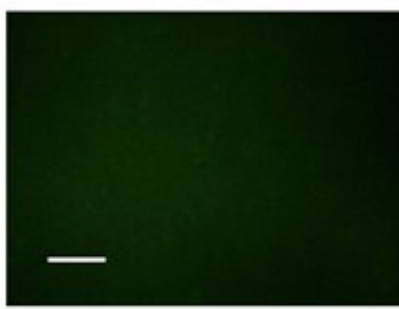

$\mathrm{F}$

Fig. 1. Identification of feline herpesvirus type 1 (FHV-1) based on cytopathic effects (CPE) and fluorescence assays. CPE in CRFK cells infected with FHV191071 and FHV191072 isolates at $48 \mathrm{~h}$ post-inoculation (A, B); specific fluorescence observed in CRFK cells infected with FHV191071 and FHV191072 (D, E); and normal CRFK cells (C and F). Six pictures are magnified 200 times and scale bars indicate $100 \mu \mathrm{m}$.

A

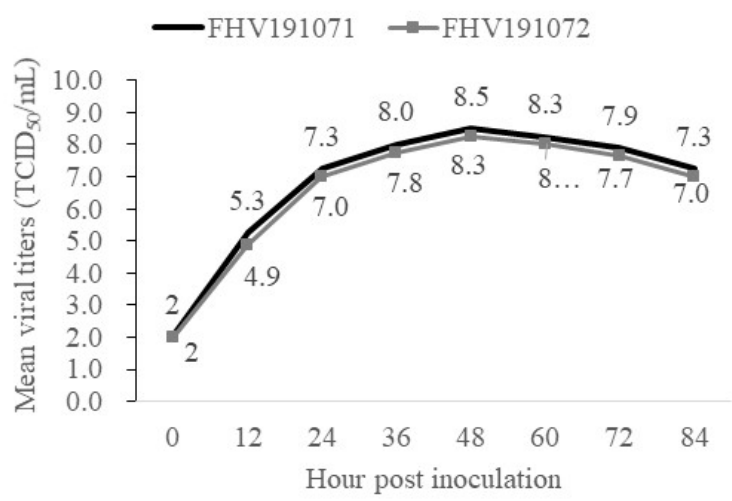

B

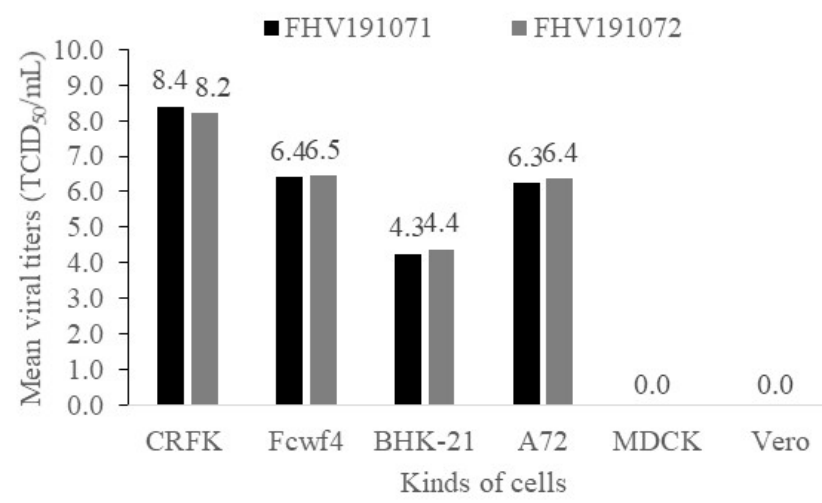

Fig. 2. Growth kinetics of FHV191071 and FHV191072 isolates, according to harvest time in CRFK cells and viral titers of two FHV isolates propagated in six cell types to examine cell tropism. The isolates showed maximum titers of $10^{8.5}$ and $10^{8.3}$ $\mathrm{TCID}_{50} / \mathrm{mL}$ in CRFK cells at $48 \mathrm{~h}$ post-inoculation (A). Mean viral titers of the two isolates ranged from $10^{4.3}$ to $10^{8.4} \mathrm{TCID} 50 / \mathrm{mL}$ in four cell types, while neither isolate grew in MDCK or Vero cells (B). 
A
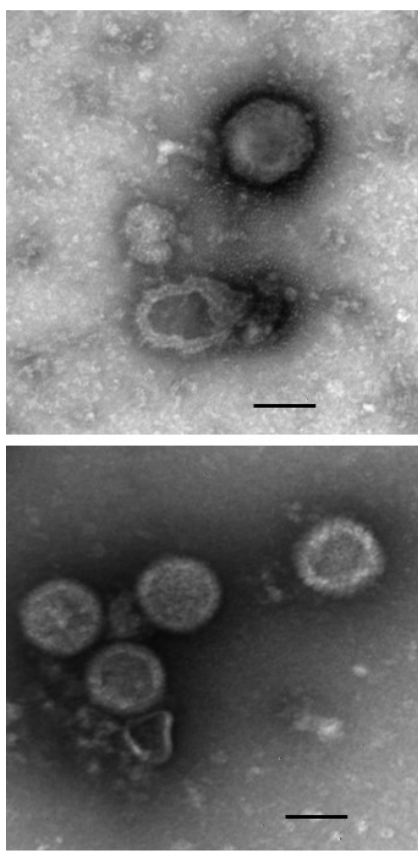

C
B
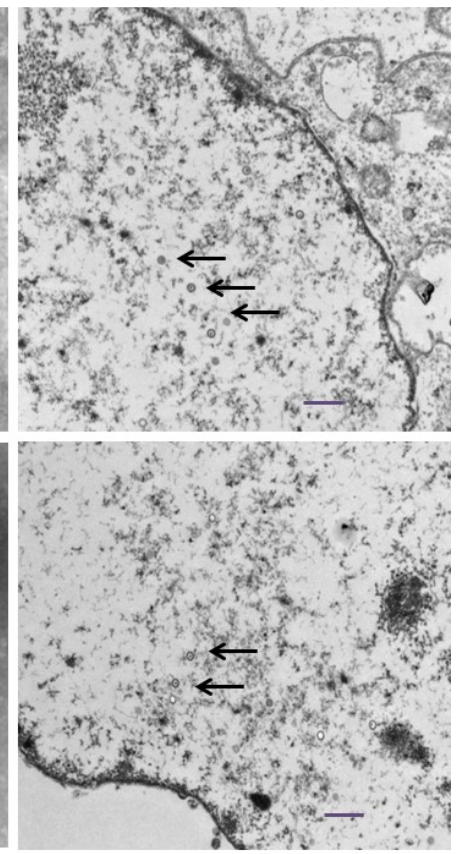

D

Fig. 3. Viral particles of FHV191071 and FHV191072 isolates purified with sucrose gradient centrifugation (A, C) and in the nuclei of infected CRFK cells, as observed by electron microscopy (B, D). Scale bars indicate $100 \mathrm{~nm}(\mathrm{~A}, \mathrm{C})$ and $500 \mathrm{~nm}(\mathrm{~B}, \mathrm{D})$.

antigens for EM were prepared by sucrose purification. Virus particles of the FHV191071 and FHV191072 isolates were identified by EM from purified solutions by sucrose density gradient ultracentrifugation of infected cells. Viral particles with pleomorphic forms were 140-170 nm in diameter with crown-shaped projections, a morphological appearance typical of Herpesviridae (Fig. 3A, C). Viral particles were also observed in the nuclei of CRFK cells infected with both isolates (Fig. 3B, D).

\section{Molecular characterization of FHV isolates}

PCR, cloning, and sequencing were used to determine the genetic features of the two isolates. As shown in Fig. 4, six PCR products from the FHV191071 isolate were detected at sizes of 519,620,670, 889, 1109, and 1194 bp on $2.0 \%$ agarose gels, confirming that the isolates were FHV-1 based on the expected size of the PCR products. The entire $T K$ and $g B$ gene sequences of FHV191071 and FHV191072 isolates comprised 1,032 and 2,847 nucleotides encoding 344 and 949 amino acids, respectively. The $T K$ and $g B$ genes were compared with those of 16 herpesviruses in GenBank to determine their genetic relationships with other herpesviruses.

The $T K$ and $g B$ genes of the FHV191071 and FHV191072 isolates had identical nucleotide sequences and shared $99.9 \%$ to $100 \%$ nucleotide homology with those of other feline herpesviruses (e.g., KANS-02, KANA-04, and 729-83 strains reported in the USA and Australia) (11). Phylogenetic trees were constructed based on the two genes in 16 herpesviruses to examine the genetic relationships of the two isolates. As shown in Fig. 5, the herpesviruses were subdivided according to host animal species (i.e., swine, bovine, equine, feline, and canine); both isolates belonged to the feline group. The full amino acid sequences of the TK genes of FHV191071 and FHV1901072 isolates and the KANS-02 strain were aligned to examine genetic features of the two isolates. As shown in Fig. 6, one potential $\mathrm{N}$-linked glycosylation site ( $\mathrm{N}-\mathrm{X}-\mathrm{S} / \mathrm{T}$ ) was identified in the TK protein; one amino acid (isoleucine) was replaced by threonine at position 341 in the TK protein of both isolates. 


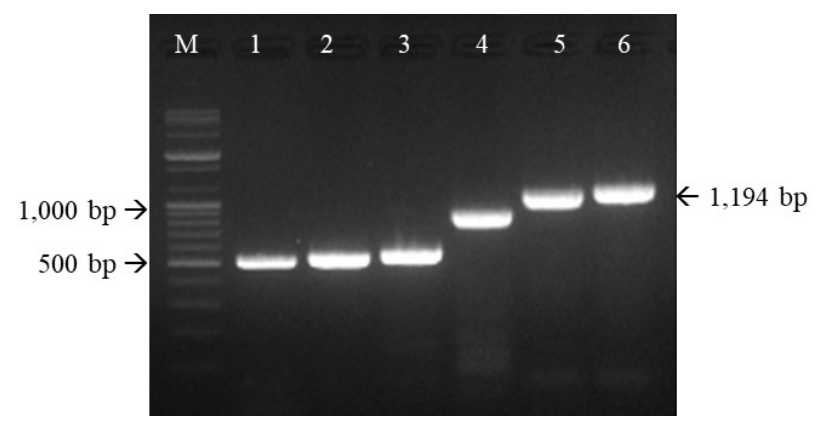

Fig. 4. Six primer sets targeting the thymidine kinase $(T K)$ and glycoprotein $B(g B)$ genes of the FHV191071 isolate were used for polymerase chain reaction (PCR). PCR products of expected sizes confirmed that the FHV191071 isolate was feline herpesvirus type 1. M, 1 kb ladder; PCR products of the TK (lanes 1-3) and $g B$ (lanes 4-6) genes of the FHV191071 isolate.

A

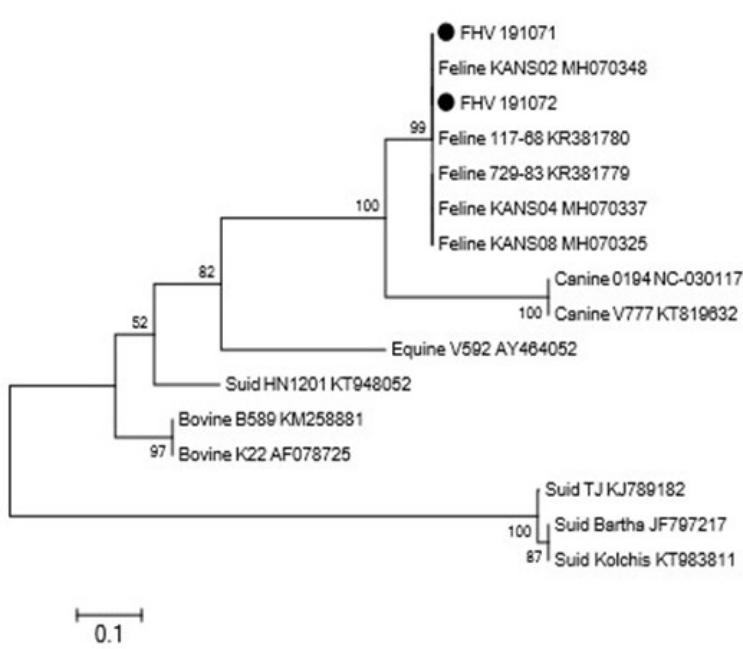

B

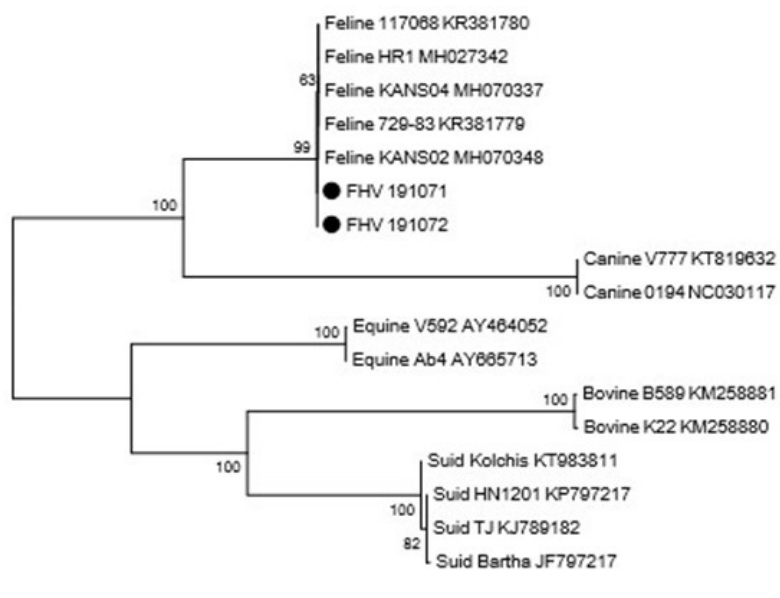

0.1

Fig. 5. Phylogenetic trees based on the thymidine kinase $(T K)(A)$ and glycoprotein $B(g B)$ genes of animal herpesviruses. In both phylogenetic trees, the FHV191071 and FHV191072 isolates belong to the feline herpesvirus type 1 group.

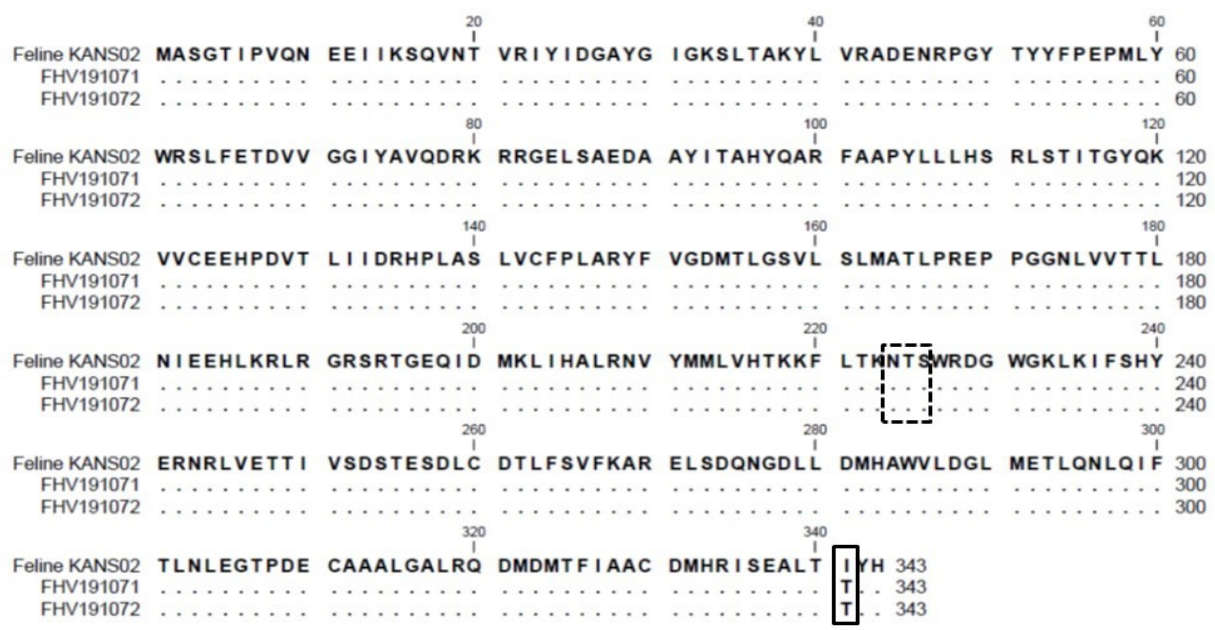

Fig. 6. Alignment of amino acid sequences of TK proteins of the FHV191071 and FHV191072 isolates and the KANS-02 strain. The amino acid threonine at position 341 in the TK protein of the Korean FHV isolates was replaced by isoleucine (solid box). A potential $\mathrm{N}$-linked glycosylation site $(\mathrm{N}-\mathrm{X}-\mathrm{S} / \mathrm{T})$ is indicated by the box with a dashed line. 


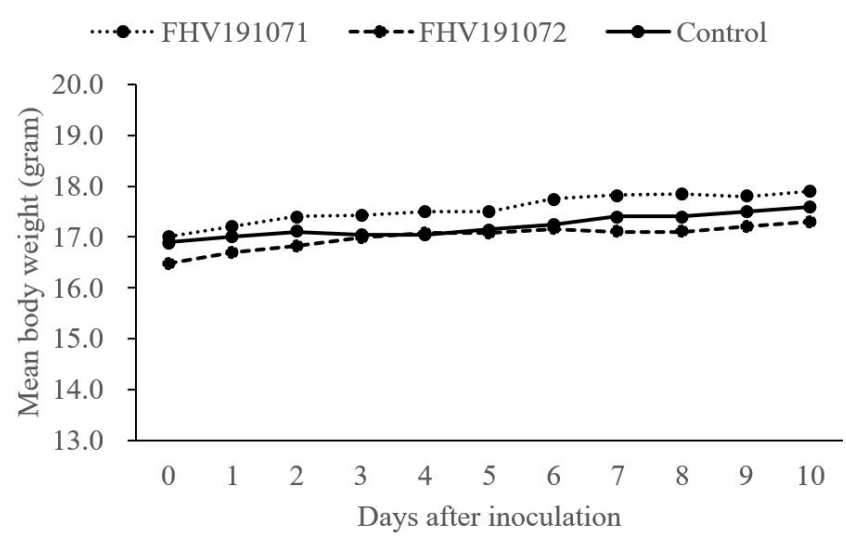

Fig. 7. Changes in mean body weight in mice inoculated with FHV191071 and FHV191072 isolates intraperitoneally. No mice lost weight during the 10-day experimental protocol.

\section{Virulence of two isolates in mice}

Six 4-week-old Balb/c mice were inoculated with FHV191071 and FHV191072 isolates at viral titers of $10^{8.0} \mathrm{TCID}_{50} / \mathrm{mL}$ to examine the virulence characteristics of the two isolates. None of the isolate-treated mice showed clinical signs or significant weight change during the observation period (Fig. 7).

\section{DISCUSSION}

The global distribution of FHV-1 has led to reports of FHV-1 companion cats and wild cats, including tigers, cheetahs, and lions $(6,7,8,15)$. Kittens infected with FHV-1 may die or exhibit severe clinical symptoms if they do not have maternal antibodies or are immunized with a vaccine that contains FHV-1 antigen $(1,5)$. Compared with other cat diseases, it is easy to diagnose FHV-1 in cats. Nevertheless, there have been few reports regarding the prevalence of FHV-1 in Korean cats or isolation of FHV-1 from these cats (16). From 12 dead cats sent to the APQA for diagnosis in 2019, we prepared 48 samples (kidney, brain, spleen, lymph nodes, lung, heart, liver, and intestine); we isolated two causative agents from kidney and brain homogenates of two cats, and cultured them in CRFK cells. These isolates were designated FHV191071 and FHV191072, respectively. We confirmed that both isolates were FHV-1 based on specific cell CPE, immunofluorescent assay, EM, PCR, and nucleotide sequence analysis. We attempted to identify histological lesions in the diagnosed cats by hematoxylin and eosin staining; however, no typical FHV-1 lesions were present. This may be early infection or latent infection. The most common laboratory diagnostic methods for FHV-1 are virus isolation and PCR using oronasal and conjunctival swabs, which yield DNA extracts $(15,17)$. Therefore, it is preferable to apply diagnostic methods including virus isolation and PCR to oronasal discharges of animals with suspected infection.

In this study, we found that CRFK cells produced the highest titers of the FHV-1 isolates among six cell lines, while MDCK and Vero cells showed no growth. Viral titers of the isolates reached $10^{8.5}$ and $10^{8.3} \mathrm{TCID}_{50} / \mathrm{mL} 48$ after inoculation, similar to wild-type FHV-1, C-27 strain (ATCC, Manassas, VA, USA) (2); this finding implied that the FHV191071 and FHV191072 isolates constituted wild-type virus circulating in stray cats. EM analysis of purified virus and infected CRFK cells revealed typical herpesvirus morphology. FHV191071 and FHV191072 particles were similar in size and shape to those of herpesviruses isolated from animals $(15,18)$. PCR detection methods (e.g., real-time and priming isothermal amplification assays) have previously been used to detect FHV-1 $(4,12)$. PCR amplification of FHV-1 based on the TK and $g B$ genes yielded bands of the expected size, which confirmed the identity of FHV-1. 
Phylogenetic analyses of FHV-1 $T K$ and $g B$ genes have been used to study evolutionary relationships and identify genetic variation among herpesviruses (1). When the $T K$ genes of 16 herpesviruses were compared, the $T K$ genes of both isolates were very similar to those of FHV-1. Sequence analyses of the $g B$ genes of 15 herpesviruses, including both isolates, revealed that both isolates were in the FHV-1 group and exhibited comparable similarity to $g B$ in other FHV-1 strains (e.g., KANS-02 and HR1). The reported genetic distance among FHV-1 stains is very low $(0.093 \%)$ and the $T K$ and $g B$ genes are highly conserved within Varicellovirus $(1,15)$; the results regarding Korean FHV-1 isolates were consistent with previous results.

It has been well known that TK gene of Alphaherpesviruses is one of virulence factors and TK proteins are highly divergent. Alignment of the full amino acid sequences of TK proteins from both isolates with the same region from the KANS-02 strain revealed only one amino acid difference among the three FHV-1s, at position 341. Nunberg et al. reported that the TK gene was nonessential for herpesvirus growth and that loss of TK activity was involved in the attenuation of herpesvirus (9). The loss of function of the TK or gE gene provided the concept of a differential herpesvirus vaccine. Thus, FHV-1 with missing or mutated parts of the TK gene can be used as a strain marker. The isolation of FHV-1 from naturally infected cats is the first step in the development of a new FHV-1 vaccine, using FHV-1 isolated from Korean cats. A future study should sequence the entire genome of the Korean FHV-1 isolates or passaged virus.

In a mouse model, the Korean FHV-1 isolates did not cause any clinical signs in 4-week-old mice inoculated peritoneally, implying that factors associated with the alpha/beta interferon system are responsible for the replication of FHV-1 in mice. Bovine herpesvirus 1 and 5 can cause neurovirulence in interferon receptor-deficient mice (19). We found no clinical evidence that the FHV191071 and FHV191072 isolates were virulent in 4-week-old mice. Therefore, further studies regarding the virulence and immunogenicity of the Korean isolates are required in target animals, such as cats.

In conclusion, we isolated two virfuses from naturally infected cats using CRFK cells and confirmed that they were FHV-1 closely related to the KANS-02 strain, which has been reported in the USA. CRFK cells produced the highest titers of wild FHV-1. The FHV191071 and FHV-1.91-07.2 isolates may provide raw material for use in developing a new FHV-1 vaccine and diagnostic reagents.

\section{ACKNOWLEDGEMENTS}

This study was supported financially by a grant (B-1543083-2020-22-01) from the Animal and Plant Quarantine Agency, Ministry of Agriculture, Food and Rural Affairs (MAFRA), Korea.

\section{REFERENCES}

1) Lewin AC, Kolb AW, McLellan GJ, Bentley E, Bernard KA, Newbury SP, et al. Genomic, Recombinational and Phylogenetic Characterization of Global Feline Herpesvirus 1 Isolates. Virology 2018;518:385-97.

2) Tai SHS, Niikura M, Cheng HH, Kruger JM, Wise AG, Maes RK. Complete genomic sequence and an infectious BAC clone of feline herpesvirus-1 (FHV-1). Virology 2010;401:215-27.

3) Gaskell R, Dawson S, Radford A, Thiry E. Feline herpesvirus. Vet Res 2007:38:337-54.

4) Tan Y, Dong G, Xu H, Niu J, Lu W, Wang K, et al. Development of a cross-priming isothermal amplification assay based on the glycoprotein $\mathrm{B}$ gene for instant and rapid detection of feline herpesvirus type 1. Arch Virol 2020;165:743-7. 
5) Lee Y, Maes R, Tai SS, Soboll Hussey G. Viral replication and innate immunity of feline herpesvirus-1 virulence-associated genes in feline respiratory epithelial cells. Virus Res 2019;264:56-67.

6) Harbour DA, Howard PE, Gaskell RM. Isolation of feline calicivirus and feline herpesvirus from domestic cats 1980 to 1989. Vet Rec 1991;128:77-80.

7) Henzel A, Brum MCS, Lautert C, Martins M, Lovato LT, Weiblen R. Isolation and identification of feline calicivirus and feline herpesvirus in Southern Brazil. Braz J Microbio/2012;43:560-8.

8) Hora AS, Tonietti PO, Guerra JM, Leme MC, Pena HFJ, Maiorka PC, et al. Felid herpesvirus 1 as a causative agent of severe nonsuppurative meningoencephalitis in a domestic cat. J Clin Microbio/2013:51:676-9.

9) Nunberg JH, Wright DK, Cole GE, Petrovskis EA, Post LE, Compton T, et al. Identification of the thymidine kinase gene of feline herpesvirus: use of degenerate oligonucleotides in the polymerase chain reaction to isolate herpesvirus gene homologs. J Virol 1989;63:3240-9.

10) Gould D. Feline herpesvirus-1: ocular manifestations, diagnosis and treatment options. J Feline Med Surg 2011;13:333-46.

11) Maes R. Felid herpesvirus type 1 infection in cats: a natural host model for alphaherpesvirus pathogenesis. ISRN Vet Sci2012:2012:495830.

12) Helps C, Reeves N, Egan K, Howard P, Harbour D. Detection of Chlamydophila felis and feline herpesvirus by multiplex real-time PCR analysis. J Clin Microbio/2003;41:2734-6.

13) Litster A, Wu CC, Leutenegger CM. Detection of feline upper respiratory tract disease pathogens using a commercially available real-time PCR test. Vet J 2015;206:149-53.

14) Yagami K, Furukawa T, Fukui M. Serologic and virologic surveys on feline herpesvirus and feline calicivirus infections in cats for experimental use. Jikken Dobutsu 1985;34:241-8.

15) Sun H, Li Y, Jiao W, Liu C, Liu X, Wang H, et al. Isolation and identification of feline herpesvirus type 1 from a South China tiger in China. Viruses 2014;6:1004-14.

16) Kang BT, Park HM. Prevalence of feline herpesvirus 1, feline calicivirus and Chlamydophila felis in clinically normal cats at a Korean animal shelter. J Vet Sci2008;9:207-9.

17) Liu MZ, Han XH, Yao LQ, Zhang WK, Liu BS, Chen ZL. Development and application of a simple recombinase polymerase amplification assay for rapid point-of-care detection of feline herpesvirus type 1. Arch Virol 2019;164:195-200.

18) Hoferer M, Braun A, Sting R. Creation of a bovine herpes virus 1 (BoHV-1) quantitative particle standard by transmission electron microscopy and comparison with established standards for use in real-time PCR. Biologicals 2017:48:121-5.

19) Abril C, Engels M, Liman A, Hilbe M, Albini S, Franchini M, et al. Both viral and host factors contribute to neurovirulence of bovine herpesviruses 1 and 5 in interferon receptor-deficient mice. J Viro/2004;78:3644-53. 